\title{
Ramsey-Turán numbers for semi-algebraic graphs
}

\author{
Jacob Fox* \\ Department of Mathematics \\ Stanford University \\ Stanford, CA, U.S.A. \\ jacobfox@stanford.edu
}

\author{
János $\mathrm{Pach}^{\dagger}$ \\ Chair of Combinatorial Geometry DCG \\ École Polytechnique Fédérale de Lausanne \\ Lausanne, Switzerland \\ pach@cims.nyu.edu
}

\author{
Andrew Suk \\ Department of Mathematics \\ University of California San Diego \\ La Jolla, CA, U.S.A. \\ asuk@ucsd.edu
}

Submitted: Jul 7, 2018; Accepted: Nov 26, 2018; Published: Dec 21, 2018

(C) The authors. Released under the CC BY-ND license (International 4.0).

\begin{abstract}
A semi-algebraic graph $G=(V, E)$ is a graph where the vertices are points in $\mathbb{R}^{d}$, and the edge set $E$ is defined by a semi-algebraic relation of constant complexity on $V$. In this note, we establish the following Ramsey-Turán theorem: for every integer $p \geqslant 3$, every $K_{p}$-free semi-algebraic graph on $n$ vertices with independence number $o(n)$ has at most $\frac{1}{2}\left(1-\frac{1}{\lceil p / 2\rceil-1}+o(1)\right) n^{2}$ edges. Here, the dependence on the complexity of the semi-algebraic relation is hidden in the $o(1)$ term. Moreover, we show that this bound is tight.
\end{abstract}

Mathematics Subject Classifications: 05D10, 52C10

\section{Introduction}

Over the past decade, several authors have shown that many classical theorems in extremal graph theory can be significantly improved if we restrict our attention to semi-algebraic graphs, that is, graphs whose vertices are points in Euclidean space, and edges are defined by a semi-algebraic relation of constant complexity $[1,5,8,11,9,4]$. In this note, we

*Supported by a Packard Fellowship and by NSF CAREER award DMS 1352121.

${ }^{\dagger}$ Supported by Swiss National Science Foundation Grants 200020-162884 and 200021-175977.

${ }^{\ddagger}$ Supported an NSF CAREER award and an Alfred Sloan Fellowship. 
continue this sequence of works by studying Ramsey-Turán numbers for semi-algebraic graphs.

More formally, a graph $G=(V, E)$ is a semi-algebraic graph with complexity at most $t$, if its vertex set $V$ is an ordered set of points in $\mathbb{R}^{d}$, where $d \leqslant t$, and if there are at most $t$ polynomials $g_{1}, \ldots, g_{s} \in \mathbb{R}\left[x_{1}, \ldots, x_{2 d}\right], s \leqslant t$, of degree at most $t$ and a Boolean formula $\Phi$ such that for vertices $u, v \in V$ such that $u$ comes before $v$ in the ordering,

$$
(u, v) \in E \quad \Leftrightarrow \quad \Phi\left(g_{1}(u, v) \geqslant 0 ; \ldots ; g_{s}(u, v) \geqslant 0\right)=1 .
$$

At the evaluation of $g_{\ell}(u, v)$, we substitute the variables $x_{1}, \ldots, x_{d}$ with the coordinates of $u$, and the variables $x_{d+1}, \ldots, x_{2 d}$ with the coordinates of $v$. Here, we assume that the complexity $t$ is a fixed parameter, and $n=|V|$ tends to infinity.

The classical theorem of Turán gives the maximum number of edges in a $K_{p}$-free graph on $n$ vertices.

Theorem 1 (Turán, [13]). Let $G=(V, E)$ be a $K_{p}$-free graph with $n$ vertices. Then

$$
|E| \leqslant \frac{1}{2}\left(1-\frac{1}{p-1}+o(1)\right) n^{2} .
$$

The only graph for which this bound is tight is the complete $(p-1)$-partite graph whose parts are of size as equal as possible. This graph can easily be realized as an intersection graph of segments in the plane, which is a semi-algebraic graph with complexity at most four. Therefore, Turán's theorem cannot be improved by restricting it to semi-algebraic graphs.

Let $H$ be a fixed graph. The Ramsey-Turán number $\mathbf{R T}(n, H, \alpha)$ is defined as the maximum number of edges that an $n$-vertex graph of independence number at most $\alpha$ can have without containing $H$ as a (not necessarily induced) subgraph. Ramsey-Turán numbers were introduced by Andrásfai [2] and were motivated by the classical theorems of Ramsey and Turán and their connections to geometry, analysis, and number theory. According to one of the earliest results in Ramsey-Turán theory, which appeared in [7], for every $p \geqslant 2$, we have

$$
\mathbf{R T}\left(n, K_{2 p-1}, o(n)\right)=\frac{1}{2}\left(1-\frac{1}{p-1}\right) n^{2}+o\left(n^{2}\right) .
$$

For excluded $K_{4}$, a celebrated result of Szemerédi [12] and Bollobás-Erdős [3] states that

$$
\mathbf{R T}\left(n, K_{4}, o(n)\right)=\frac{1}{8} n^{2}+o\left(n^{2}\right) .
$$

This was generalized by Erdős, Hajnal, Sós, and Szemerédi [6] to all cliques of even size. For every $p \geqslant 2$, we have

$$
\mathbf{R T}\left(n, K_{2 p}, o(n)\right)=\frac{1}{2} \cdot \frac{3 p-5}{3 p-2} n^{2}+o\left(n^{2}\right) .
$$


For more results in Ramsey-Turán theory, consult the survey of Simonovits and Sós [10].

In the present note, we establish asymptotically tight bounds on Ramsey-Turán numbers for semi-algebraic graphs. We define $\mathbf{R T}_{t}\left(n, K_{p}, o(n)\right)$ as the maximum number of edges that $n$-vertex $K_{p}$-free semi-algebraic graphs with complexity at most $t$ can have, if their independence number is $o(n)$. Strictly speaking, this definition and all above results apply to sequences of graphs with $n$ vertices, as $n$ tends to infinity.

It turns out that if the size of the excluded clique is even, then the answer to the Ramsey-Turán question significantly changes when the graphs are required to be semialgebraic. However, in the odd case, we obtain the same asymptotics for the RamseyTurán function as in (1). More precisely, we have

Theorem 2. For any fixed integers $t \geqslant 5$ and $p \geqslant 2$, we have

$$
\mathbf{R T}_{t}\left(n, K_{2 p-1}, o(n)\right)=\mathbf{R T}_{t}\left(n, K_{2 p}, o(n)\right)=\frac{1}{2}\left(1-\frac{1}{p-1}\right) n^{2}+o\left(n^{2}\right)
$$

\section{Proof of Theorem 2}

The aim of this section is to prove Theorem 2. One of the main tools used in the proof is the following regularity lemma for semi-algebraic graphs. Given a graph $G=(V, E)$, a vertex partition is called equitable if any two parts differ in size by at most one. Given two disjoint subsets $V_{i}, V_{j} \subset V$, we say that the pair $\left(V_{i}, V_{j}\right)$ is homogeneous if $V_{i} \times V_{j} \subset E$ or $\left(V_{i} \times V_{j}\right) \cap E=\emptyset$.

Lemma $3([9])$. For any positive integer $t$, there exists a constant $c=c(t)>0$ with the following property. Let $0<\varepsilon<1 / 2$ and let $G=(V, E)$ be a semi-algebraic graph with complexity at most $t$. Then $V$ has an equitable partition $V=V_{1} \cup \cdots \cup V_{K}$ into $K$ part, where $1 / \varepsilon<K<(1 / \varepsilon)^{c}$, such that all but an $\varepsilon$-fraction of the pairs of parts are homogeneous.

The upper bound in Theorem 2 follows from

Theorem 4. Let $\varepsilon>0$ and let $G=(V, E)$ be an $n$-vertex semi-algebraic graph with complexity at most $t$. If $G$ is $K_{2 p}$-free and $|E|>\frac{1}{2}\left(1-\frac{1}{p-1}+\varepsilon\right) n^{2}$, then $G$ has an independent set of size $\gamma n$, where $\gamma=\gamma(t, p, \varepsilon)$.

Proof. We apply Lemma 3 with parameter $\varepsilon / 4$ to obtain an equitable partition $\mathcal{P}: V=$ $V_{1} \cup \cdots \cup V_{K}$ such that $\frac{4}{\varepsilon} \leqslant K \leqslant\left(\frac{4}{\varepsilon}\right)^{c}$, where $c=c(t)$ and all but an at most $\frac{\varepsilon}{4}$-fraction of all pairs of parts in $\mathcal{P}$ are homogeneous (complete or empty with respect to $E$ ). If $n \leqslant 10 K$, then $G$ has an independent set of size one, and the theorem holds trivially. So, we may assume $n>10 K$.

By deleting all edges inside each part, we have deleted at most

$$
K\left(\begin{array}{c}
\lceil n / K\rceil \\
2
\end{array}\right) \leqslant \frac{4 n^{2}}{5 K} \leqslant \varepsilon \frac{n^{2}}{5}
$$


edges. Deleting all edges between non-homogeneous pairs of parts, we lose an additional at most

$$
\left\lceil\frac{n}{K}\right\rceil^{2} \frac{\varepsilon}{4} \frac{K^{2}}{2} \leqslant \varepsilon \frac{n^{2}}{5}
$$

edges. In total, we have deleted at most $2 \varepsilon n^{2} / 5$ edges of $G$. The only edges that remain in $G$ are edges between homogeneous pairs of parts, and we have at least $\frac{1}{2}\left(1-\frac{1}{p-1}+\varepsilon / 5\right) n^{2}$ edges. By Turán's theorem (Theorem 1), there is at least one remaining copy of $K_{p}$, and its vertices lie in $p$ distinct parts $V_{i_{1}}, \ldots, V_{i_{p}} \in \mathcal{P}$ that form a complete $p$-partite subgraph. If any of the parts $V_{i_{j}}$ forms an independent set in $G$, then there is an independent set of order $\left|V_{i_{j}}\right| \geqslant\lfloor n / K\rfloor \geqslant \gamma n$, where $\gamma=\gamma(t, \epsilon, p)$, and we are done. Otherwise, there is an edge in each of the $p$ parts, and the endpoints of these $p$ edges form a $K_{2 p}$ in $G$, a contradiction.

The lower bound on $\mathbf{R T}\left(n, K_{2 p-1}, o(n)\right)$ and $\mathbf{R T}\left(n, K_{2 p}, o(n)\right)$ in Theorem 2 is constructive and is based on the following result of Walczak.

Lemma 5 ([14]). For any pair of positive integers $n$ and $p$, where $n$ is a multiple of $p-1$, there is a collection $S$ of $n /(p-1)$ segments in the plane whose intersection graph $G_{S}$ is triangle-free and has no independent set of size $c_{p} n / \log \log n$. Here $c_{p}$ is a suitable constant.

The construction. Take $p-1$ dilated copies of a set $S$ meeting the requirements in Lemma 5 , and label them as $S_{1}, \ldots, S_{p-1}$, so that $S_{i}$ lies inside a ball with center $(i, 0)$ and radius $1 / 10$. Set $V=S_{1} \cup \cdots \cup S_{p-1}$. Note that $\left|S_{i}\right|=n /(p-1)$ so that $|V|=n$. Let $G=(V, E)$ be the graph whose vertices are the elements of $V$, and two vertices (that is, two segments) are connected by an edge if and only if they cross or their left endpoints are at least $1 / 2$ apart. The graph $G$ consists of a complete $(p-1)$-partite graph, where each part induces a copy of the triangle-free graph $G_{S}$. Clearly, $G$ is $K_{2 p-1}$-free and does not contain any independent set of size $c_{p} n / \log \log n$. Moreover,

$$
|E(G)| \geqslant \frac{1}{2}\left(1-\frac{1}{p-1}\right) n^{2} .
$$

Every segment can be represented by a point in $\mathbb{R}^{4}$, and whether or not two segments intersect can be determined by four polynomial inequalities of degree at most two (see [1]). Thus, counting the distance condition, we have 5 quadratic inequalities, showing that $E$ is a semi-algebraic relation of complexity 5 .

\section{References}

[1] N. Alon, J. Pach, R. Pinchasi, R. Radoičić, and M. Sharir, Crossing patterns of semi-algebraic sets, J. Combin. Theory Ser. A 111 (2005), 310-326.

[2] B. Andrásfai, Über ein Extremalproblem der Graphentheorie, Acta Math. Hungar. 13 (1962) 443-455. 
[3] B. Bollobás and P. Erdős, On a Ramsey-Turán type problem, J. Combin. Theory Ser. B 21 (1976), 166-168.

[4] B. Bukh, J. Matoušek, Erdős-Szekeres-type statements: Ramsey function and decidability in dimension 1, Duke J. Math. 163 (2014), 2243-2270.

[5] D. Conlon, J. Fox, J. Pach, B. Sudakov, A. Suk, Ramsey-type results for semialgebraic relations, Trans. Amer. Math. Soc. 366 (2014), 5043-5065.

[6] P. Erdős, A. Hajnal, V. Sós, and E. Szemerédi, More results on Ramsey-Turán type problems, Combinatorica 3 (1983), 69-81.

[7] P. Erdős and V. Sós, Some remarks on Ramsey's and Turan's theorem, Coll. Math. Soc. J. Bolyai 4, Comb. Theory and its Appl., North-Holland (1969), 395-404.

[8] J. Fox, J. Pach, A. Sheffer, A. Suk, J. Zahl, A semi-algebraic version of Zarankiewicz's problem, J. Eur. Math. Soc. 19 (2017), 1785-1810.

[9] J. Fox, J. Pach, and A. Suk, A polynomial regularity lemma for semi-algebraic hypergraphs and its applications in geometry and property testing, SIAM J. Comput. 45 (2016), 2199-2223.

[10] M. Simonovits and V.T. Sós, Ramsey-Turán theory, Discrete Math. 229 (2001), 293340 .

[11] A. Suk, Semi-algebraic Ramsey numbers, J. Combin. Theory Ser. B 116 (2016), 465-483.

[12] E. Szemerédi, On graphs containing no complete subgraph with 4 vertices (Hungarian), Mat. Lapok 23 (1972), 113-116.

[13] P. Turán, On an extremal problem in graph theory, Matematikai és Fizikai Lapok (in Hungarian) 48 (1941), 436-452.

[14] B. Walczak, Triangle-free geometric intersection graphs with no large independent sets, Discrete Comput. Geom. 53 (2015), 221-225. 